\title{
15. VELOCITIES, DENSITIES, AND ELASTIC CONSTANTS OF BASALT AND TRACHYTIC TUFF, DSDP LEG 39
}

\author{
R.L. Carlson and N.I. Christensen, Department of Geological Sciences, \\ University of Washington, Seattle, Washington
}

\section{INTRODUCTION}

Compressional and shear wave velocities have been measured to hydrostatic confining pressures of $6 \mathrm{kbar}$ for seven basalt samples from Sites 354 and 355 and three welded trachytic tuffs from Site 359 . The two samples selected from Site 354 are rather coarsegrained basalts with intergranular to subophitic textures. Of significance for the velocity measurements are the large amounts of calcite $(10 \%$ to $20 \%)$ in these samples occurring as groundmass alteration, amygdules, and veins. The five basalt samples selected for velocity measurements from Site 355 are much finer grained than those from Site 354. Alteration, which is largely confined to the groundmass, is somewhat variable as is suggested by the range of bulk densities of the specimens $(2.76$ to $2.88 \mathrm{~g} / \mathrm{cc})$. The trachytic tuffs from the Walvis Ridge (Site 359) are of particular interest, because elastic properties have not been previously determined for oceanic rocks of this composition. The three trachytic samples selected for velocity measurements increase in density with depth, the increase being due to induration and hence reduction in porosity. The tuffs are composed of alkali feldspar phenocrysts and pumice fragments in a partly devitrified matrix. Detailed petrographic descriptions of the samples from these three sites are given elsewhere in this volume.

\section{EXPERIMENTAL TECHNIQUE AND DATA}

Compressional $\left(V_{p}\right)$ and shear $\left(V_{s}\right)$ velocities (Table 1) were measured for each specimen using the pulse transmission technique described by Birch (1960). The core diameters of the specimens were $2.54 \mathrm{~cm}$ and the lengths ranged from 2.9 to $3.6 \mathrm{~cm}$. Compressional and shear waves were generated by $2-\mathrm{MHz}$ barium titanate and $\mathrm{AC}$ cut quartz transducers. The samples were water saturated prior to the measurements, and copper screens were placed between the samples and the copper jackets so that pore pressures remained minimal during the pressure runs (Christensen and Salisbury, 1975). Velocity measurements were made in increasing and decreasing pressure cycles to $6 \mathrm{kbar}$. As has been observed in many earlier investigations, there is usually a slight hysteresis in velocity which is most pronounced at pressures below 1 or $2 \mathrm{kbar}$. The velocities in Table 1 are averages of the velocities recorded at increasing and decreasing pressure.

The compressional and shear wave velocities are estimated to be accurate to $0.5 \%$ and $1 \%$, respectively
(Christensen and Shaw, 1970). The pressures, which are accurate to $1 \%$, were determined by measuring the change in electrical resistance of a calibrated manganin coil. The densities recorded in Table 1 were determined from the weights and volumes of the rock cores used for the velocity measurements and thus are bulk densities.

The ratio of compressional to shear velocity $\left(V_{p} / V_{s}\right)$, Poisson's ratio $(\sigma)$, the bulk modulus $(K)$, the shear modulus $(\mu)$, Young's modulus $(E)$, and Lamé's constant $(\lambda)$ calculated from velocities and densities corrected for dimension changes (Christensen and Shaw, 1970) are given in Table 2 for selected pressures.

\section{DISCUSSION}

In Figure 1 compressional wave velocities, shear wave velocities, and densities at $0.5 \mathrm{kbar}$ for the basalts from Sites 354 and 355 and the trachytic tuffs from Site 359 are compared to similar basalt measurements from DSDP sites summarized by Christensen and Salisbury (1975). The basalts have densities near $3.0 \mathrm{~g} / \mathrm{cc}$ and compressional and shear wave velocities of $0.5 \mathrm{kbar}$ of about 6.0 and $3.6 \mathrm{~km} / \mathrm{sec}$, respectively. Though many factors affect sonic velocities, the near-linear increase of velocity with increasing density apparent in Figure 1 is primarily related to the degree of weathering and hence to the ages of the basalts. Due to alteration, the basalts from Sites 354 and 355 have densities 5\% to $10 \%$ lower than the density of fresh basalt, and the velocities are correspondingly lower. It is significant that the samples from Site 354 have higher velocities than basalts of similar density. This difference in velocity is apparently related to the high calcite content of the two samples from Site 354 because calcite has anomalously high velocities (Birch, 1960; Christensen, 1965).

As expected, the trachytic tuffs have velocities which increase with increasing density, the increase being related to decreasing porosity rather than a reduction in the amount of alteration. Figure 1 also illustrates that the velocities of the tuffs fall above velocity-density curves determined for basalts. Birch (1961) found that velocity is related to mean atomic weight as well as density, with rocks of lower mean atomic weight having higher velocities for a given density. Since tuffs have mean atomic weights which are lower than basalts (Birch, 1961), their velocities should be higher than basalts of similar densities, which is observed in Figure 1.

Seismic refraction data have not been published in the vicinities of Sites 354,355 , and 359 . Thus it is not 
TABLE 1

Compressional (P) and Shear (S) Wave Velocities

\begin{tabular}{llccccccccc}
\hline $\begin{array}{l}\text { Sample } \\
\text { (Interval in cm) }\end{array}$ & \multirow{2}{*}{$\begin{array}{c}\text { Bulk } \\
\text { Density }\end{array}$} & Mode & 0.2 & 0.4 & 0.6 & 0.8 & 1.0 & 2.0 & 4.0 & 6.0 \\
\hline $354-19-3$, & 2.733 & P & 5.55 & 5.57 & 5.59 & 5.61 & 5.62 & 5.67 & 5.74 & 5.80 \\
$131-134$ & & S & 3.03 & 3.04 & 3.05 & 3.06 & 3.06 & 3.08 & 3.10 & 3.11 \\
$354-19-5$, & 2.753 & P & 5.63 & 5.65 & 5.67 & 5.69 & 5.70 & 5.74 & 5.79 & 5.85 \\
$93-96$ & & S & 3.08 & 3.09 & 3.09 & 3.10 & 3.11 & 3.12 & 3.14 & 3.15 \\
$355-21-1$, & 2.808 & P & 5.70 & 5.71 & 5.72 & 5.73 & 5.74 & 5.75 & 5.80 & 5.86 \\
$147-150$ & & S & 2.93 & 2.95 & 2.96 & 2.97 & 2.98 & 3.02 & 3.07 & 3.10 \\
$355-22-1$, & 2.884 & P & 6.03 & 6.05 & 6.07 & 6.08 & 6.10 & 6.15 & 6.22 & 6.28 \\
$57-60$ & & S & 3.12 & 3.14 & 3.16 & 3.17 & 3.19 & 3.23 & 3.26 & 3.27 \\
$355-22-2$, & 2.838 & P & 5.78 & 5.83 & 5.86 & 5.88 & 5.89 & 5.94 & 5.99 & 6.07 \\
$44-47$ & & S & 3.20 & 3.21 & 3.22 & 3.23 & 3.24 & 3.25 & 3.26 & 3.27 \\
$355-22-4$, & 2.757 & P & 5.35 & 5.41 & 5.45 & 5.48 & 5.50 & 5.55 & 5.60 & 5.64 \\
$69-72$ & & S & 2.93 & 2.94 & 2.95 & 2.96 & 2.96 & 2.96 & 3.02 & 3.04 \\
$355-22-5$, & 2.798 & P & 5.54 & 5.57 & 5.59 & 5.61 & 5.62 & 5.67 & 5.73 & 5.81 \\
$119-122$ & & S & 3.08 & 3.09 & 3.09 & 3.10 & 3.10 & 3.12 & 3.15 & 3.17 \\
$359-4-2$, & 2.258 & P & 4.32 & 4.35 & 4.38 & 4.40 & 4.42 & 4.51 & 4.62 & 4.73 \\
$80-83^{\mathrm{a}}$ & & S & 2.04 & 2.06 & 2.10 & 2.12 & 2.14 & 2.19 & 2.23 & 2.25 \\
$359-4-3$, & 2.308 & P & 4.32 & 4.36 & 4.40 & 4.40 & 4.47 & 4.58 & 4.73 & 4.82 \\
$143-146^{\mathrm{a}}$ & & S & 2.06 & 2.09 & 2.12 & 2.14 & 2.15 & 2.19 & 2.21 & 2.24 \\
$359-5-1$, & 2.449 & P & 5.06 & 5.10 & 5.12 & 5.13 & 5.14 & 5.16 & 5.20 & 5.26 \\
$26-29^{\mathrm{a}}$ & & S & 2.45 & 2.47 & 2.48 & 2.49 & 2.50 & 2.52 & 2.54 & 2.55 \\
\hline
\end{tabular}

${ }^{\mathrm{a}}$ Tuff.

possible to compare our laboratory data with upper crustal velocities. It would be particularly interesting to have detailed refraction surveys in the vicinity of Site 359 to see if distinctions could be made between regions underlain by trachytic tuff and presumably higher density and velocity basalt.

\section{ACKNOWLEDGMENTS}

V. Kiefer, J. Hull, and K. Hubert assisted in the operation of the high pressure system. This investigation was supported by the Office of Naval Research Contract N-00014-67-A0103-0014.

\section{REFERENCES}

Birch, F., 1960. The velocity of compressional waves in rocks to 10 kilobars, 1: J. Geophys. Res., v. 65, p. 1083. 1961. The velocity of compressional waves in rocks to 10 kilobars, 2: J. Geophys. Res., v. 66, p. 2199.

Christensen, N.I., 1965. Compressional wave velocities in metamorphic rocks at pressures to $10 \mathrm{kbar}$ J. Geophys. Res., v. 70, p. 6147.

Christensen, N.I. and Salisbury, M.H., 1975. Structure and constitution of the lower oceanic crust: Rev. Geophys. Space Phys., v. 13, p. 57.

Christensen, N.I. and Shaw, G.H., 1970. Elasticity of mafic rocks from the Mid-Atlantic Ridge: Geophys. J. Roy. Astron. Soc., v. 20, p. 271. 
TABLE 2

Elastic Constants, Leg 39

\begin{tabular}{|c|c|c|c|c|c|c|c|}
\hline $\begin{array}{c}\text { Sample } \\
\text { (Interval in } \mathrm{cm} \text { ) }\end{array}$ & $\begin{array}{l}\text { Pressure } \\
\text { (kbar) }\end{array}$ & $V_{p} / V_{s}$ & $\sigma$ & $\begin{array}{c}K \\
(\mathrm{mbar})\end{array}$ & $\stackrel{\stackrel{\mu}{\mu})}{(\mathrm{mbar})}$ & $\underset{(\mathrm{mbar})}{E}$ & $\stackrel{\lambda}{\lambda}$ \\
\hline $\begin{array}{l}354-19-3, \\
131-134\end{array}$ & $\begin{array}{l}0.4 \\
1.0 \\
2.0 \\
6.0\end{array}$ & $\begin{array}{l}1.83 \\
1.84 \\
1.84 \\
1.86\end{array}$ & $\begin{array}{l}0.29 \\
0.29 \\
0.29 \\
0.30\end{array}$ & $\begin{array}{l}0.51 \\
0.52 \\
0.53 \\
0.57\end{array}$ & $\begin{array}{l}0.25 \\
0.26 \\
0.26 \\
0.27\end{array}$ & $\begin{array}{l}0.65 \\
0.66 \\
0.67 \\
0.69\end{array}$ & $\begin{array}{l}0.34 \\
0.35 \\
0.36 \\
0.39\end{array}$ \\
\hline $\begin{array}{l}354-19-5, \\
93-96\end{array}$ & $\begin{array}{l}0.4 \\
1.0 \\
2.0 \\
6.0\end{array}$ & $\begin{array}{l}1.83 \\
1.83 \\
1.84 \\
1.86\end{array}$ & $\begin{array}{l}0.29 \\
0.29 \\
0.29 \\
0.30\end{array}$ & $\begin{array}{l}0.53 \\
0.54 \\
0.55 \\
0.58\end{array}$ & $\begin{array}{l}0.26 \\
0.27 \\
0.27 \\
0.27\end{array}$ & $\begin{array}{l}0.68 \\
0.69 \\
0.69 \\
0.71\end{array}$ & $\begin{array}{l}0.35 \\
0.36 \\
0.37 \\
0.40\end{array}$ \\
\hline $\begin{array}{l}355-21-1 \\
147-150\end{array}$ & $\begin{array}{l}0.4 \\
1.0 \\
2.0 \\
6.0\end{array}$ & $\begin{array}{l}1.94 \\
1.93 \\
1.93 \\
1.89\end{array}$ & $\begin{array}{l}0.32 \\
0.32 \\
0.32 \\
0.31\end{array}$ & $\begin{array}{l}0.59 \\
0.59 \\
0.59 \\
0.59\end{array}$ & $\begin{array}{l}0.25 \\
0.25 \\
0.25 \\
0.27\end{array}$ & $\begin{array}{l}0.65 \\
0.65 \\
0.66 \\
0.69\end{array}$ & $\begin{array}{l}0.43 \\
0.43 \\
0.43 \\
0.42\end{array}$ \\
\hline $\begin{array}{l}355-22-1, \\
57-60\end{array}$ & $\begin{array}{l}0.4 \\
1.0 \\
2.0 \\
6.0\end{array}$ & $\begin{array}{l}1.93 \\
1.92 \\
1.91 \\
1.91\end{array}$ & $\begin{array}{l}0.32 \\
0.31 \\
0.31 \\
0.31\end{array}$ & $\begin{array}{l}0.68 \\
0.68 \\
0.58 \\
0.71\end{array}$ & $\begin{array}{l}0.28 \\
0.29 \\
0.29 \\
0.31\end{array}$ & $\begin{array}{l}0.75 \\
0.76 \\
0.77 \\
0.81\end{array}$ & $\begin{array}{l}0.49 \\
0.49 \\
0.49 \\
0.50\end{array}$ \\
\hline $\begin{array}{l}355-22-2 \\
44-47\end{array}$ & $\begin{array}{l}0.4 \\
1.0 \\
2.0 \\
6.0\end{array}$ & $\begin{array}{l}1.82 \\
1.82 \\
1.83 \\
1.86\end{array}$ & $\begin{array}{l}0.28 \\
0.28 \\
0.29 \\
0.30\end{array}$ & $\begin{array}{l}0.57 \\
0.59 \\
0.60 \\
0.64\end{array}$ & $\begin{array}{l}0.29 \\
0.30 \\
0.30 \\
0.30\end{array}$ & $\begin{array}{l}0.75 \\
0.76 \\
0.78 \\
0.79\end{array}$ & $\begin{array}{l}0.38 \\
0.39 \\
0.42 \\
0.44\end{array}$ \\
\hline $\begin{array}{l}355-22-4 \\
69-72\end{array}$ & $\begin{array}{l}0.4 \\
1.0 \\
2.0 \\
6.0\end{array}$ & $\begin{array}{l}1.84 \\
1.86 \\
1.88 \\
1.85\end{array}$ & $\begin{array}{l}0.29 \\
0.30 \\
0.30 \\
0.30\end{array}$ & $\begin{array}{l}0.49 \\
0.51 \\
0.53 \\
0.54\end{array}$ & $\begin{array}{l}0.24 \\
0.24 \\
0.24 \\
0.26\end{array}$ & $\begin{array}{l}0.62 \\
0.63 \\
0.63 \\
0.66\end{array}$ & $\begin{array}{l}0.33 \\
0.35 \\
0.37 \\
0.37\end{array}$ \\
\hline $\begin{array}{l}355-22-5 \\
119-122\end{array}$ & $\begin{array}{l}0.4 \\
1.0 \\
2.0 \\
6.0\end{array}$ & $\begin{array}{l}1.80 \\
1.81 \\
1.82 \\
1.83\end{array}$ & $\begin{array}{l}0.28 \\
0.28 \\
0.28 \\
0.29\end{array}$ & $\begin{array}{l}0.51 \\
0.53 \\
0.54 \\
0.57\end{array}$ & $\begin{array}{l}0.27 \\
0.27 \\
0.27 \\
0.28\end{array}$ & $\begin{array}{l}0.68 \\
0.69 \\
0.70 \\
0.73\end{array}$ & $\begin{array}{l}0.33 \\
0.35 \\
0.36 \\
0.38\end{array}$ \\
\hline $\begin{array}{l}359-4-2, \\
80-83\end{array}$ & $\begin{array}{l}0.4 \\
1.0 \\
2.0 \\
6.0\end{array}$ & $\begin{array}{l}2.11 \\
2.07 \\
2.06 \\
2.10\end{array}$ & $\begin{array}{l}0.36 \\
0.35 \\
0.35 \\
0.35\end{array}$ & $\begin{array}{l}0.30 \\
0.30 \\
0.32 \\
0.35\end{array}$ & $\begin{array}{l}0.10 \\
0.10 \\
0.11 \\
0.11\end{array}$ & $\begin{array}{l}0.26 \\
0.28 \\
0.29 \\
0.31\end{array}$ & $\begin{array}{l}0.24 \\
0.23 \\
0.24 \\
0.28\end{array}$ \\
\hline $\begin{array}{l}359-4-3, \\
143-146\end{array}$ & $\begin{array}{l}0.4 \\
1.0 \\
2.0 \\
6.0\end{array}$ & $\begin{array}{l}2.09 \\
2.08 \\
2.09 \\
2.15\end{array}$ & $\begin{array}{l}0.35 \\
0.35 \\
0.35 \\
0.36\end{array}$ & $\begin{array}{l}0.30 \\
0.32 \\
0.34 \\
0.38\end{array}$ & $\begin{array}{l}0.10 \\
0.11 \\
0.11 \\
0.12\end{array}$ & $\begin{array}{l}0.27 \\
0.29 \\
0.30 \\
0.32\end{array}$ & $\begin{array}{l}0.24 \\
0.25 \\
0.26 \\
0.31\end{array}$ \\
\hline $\begin{array}{l}359-5-1 \\
26-29\end{array}$ & $\begin{array}{l}0.4 \\
1.0 \\
2.0 \\
6.0\end{array}$ & $\begin{array}{l}2.06 \\
2.06 \\
2.05 \\
2.06\end{array}$ & $\begin{array}{l}0.35 \\
0.35 \\
0.34 \\
0.35\end{array}$ & $\begin{array}{l}0.44 \\
0.44 \\
0.45 \\
0.47\end{array}$ & $\begin{array}{l}0.15 \\
0.15 \\
0.16 \\
0.16\end{array}$ & $\begin{array}{l}0.40 \\
0.41 \\
0.42 \\
0.43\end{array}$ & $\begin{array}{l}0.34 \\
0.34 \\
0.34 \\
0.36\end{array}$ \\
\hline
\end{tabular}


R.L. CARLSON, N.I. CHRISTENSEN

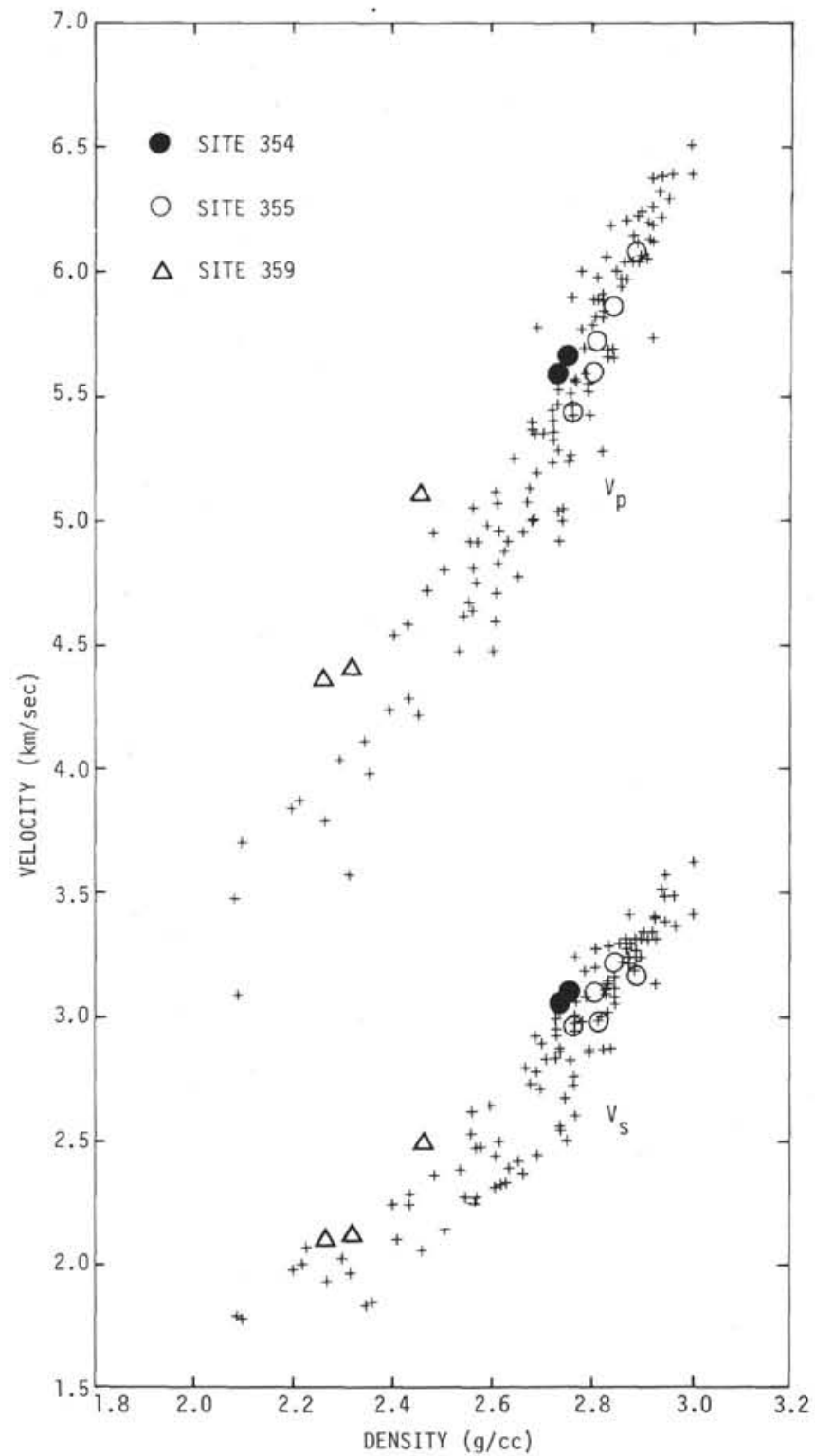

Figure 1. Density versus velocity at $0.5 \mathrm{kbar}$. Pluses represent DSDP basalt data summarized by Christensen and Salisbury (1975). 